\title{
Effect of Ozone on Apelin and APJ Expressions in Rat Peritonitis-Constituted With Colon Anastomosis
}

\section{Leyla Kilinc}

Akdeniz University: Akdeniz Universitesi https://orcid.org/0000-0002-0946-2565

\section{Sema Avci}

Alanya Alaaddin Keykubat Üniversitesi: Alanya Alaaddin Keykubat Universitesi

\section{Hakan Soylu}

Duzce University: Duzce Universitesi

\section{Tugrul Cakir}

Antalya Eğitim ve Araştırma Hastanesi: Antalya Egitim ve Arastirma Hastanesi

\section{Arif Aslaner}

Antalya Eğitim ve Araştırma Hastanesi: Antalya Egitim ve Arastirma Hastanesi

Gökhan Akkoyunlu ( $\nabla$ gokhan_akkoyunlu@hotmail.com )

Akdeniz University: Akdeniz Universitesi https://orcid.org/0000-0003-4500-8068

Ismail Ustunel

Akdeniz University: Akdeniz Universitesi

\section{Research Article}

Keywords: Colon, Anastomosis, Peritonitis, Apelin, APJ

Posted Date: August 23rd, 2021

DOI: https://doi.org/10.21203/rs.3.rs-811140/v1

License: (c) (i) This work is licensed under a Creative Commons Attribution 4.0 International License.

Read Full License 


\section{Abstract}

Background: Analyze the effect of ozone therapy on Apelin and APJ expressions in peritonitis constituted colon anastomosis.

Methods: Eighteen male Wistar albino rats weighing 250-300 g were used in this study. The rats were randomly assigned into three groups. In the colonic tissue samples Hematoxylin-Eosin staining (HE) and the Apelin and APJ immunostaining was applied. Also, Apelin and APJ protein levels between groups were determined with the Western-Blot method.

Results: In the ozone therapy group, Apelin and APJ immunoreactivity was decreased compared to the anastomosis group. The protein levels of Apelin and APJ according to Western-Blot analysis are consistent with immunostaining.

Conclusion: As a result, increased levels of Apelin and APJ in cecal punctuation and colonic anastomosis process can be deduced and said to contribute to worsening of tissue while it may be involved in return to normal of with treatment.

\section{Introduction}

The anastomosis is a surgical procedure that is performed very often in the gastrointestinal system. Several complications can occur as a consequence of this surgical procedure as anastomosis leakage. Peritonitis can be associated with these complications and can occur after a surgical procedure (Khalili et al. 2001; Cakir et al. 2016).

Medical ozone (03) is a water-soluble molecule composed of three oxygen molecules. It is applied in a variety of acute and chronic diseases as therapeutic. It is a remedy when used on the appropriate concentrations (Smith et al. 2017). Medical ozone at low concentrations down-regulates inflammation by activation of a variety of antioxidant pathways (Veneri et al. 2020). Medical ozone therapy is applied using a gas mixture composed of oxygen and ozone. Medical ozone as a mixture of 02-03 used as therapeutic purposes in many pathological processes such as peritonitis (Cakir et al. 2016; Bulynin and Glukhov 1999) and colitis (Aslaner et al. 2016). According to some researches, the beneficial effect of medical ozone used on post-surgical recovery can be achieved by various antioxidant mechanisms, by increasing tissue oxygenation, and by promoting the production of various growth factors (Erginel et al. 2014).

In previous studies, it has been reported that Apelin / APJ can regulate gastrointestinal (GI) function (Wang et al. 2004) and insulin sensitivity (Castan-Laurell et al. 2011), promote cell proliferation and migration (Lv et al. 2016). Also, it was reported that apelin messenger RNA is widely expressed in GI tissues, including the stomach and small intestine, which is closely correlated with GI function. Thus, the apelin/APJ system may exert a broad range of activities in the digestive system (18). In addition to its well-defined action, the apelin/APJ system exhibits a widespread expression pattern in the GI tract 
including enteric neurons reside in myenteric and submucosal plexus, smooth muscle cells, and gastric mucosal cells whereas enteric apelin contributes to the action of corticotropin-releasing factor (CRF) on colonic motor functions under stressed conditions (19).

Previous studies demonstrated that peripherally administered apelin-13 inhibits GI tissue motor functions (Bulbul et al. 2017), apelin-13 can reduce cell turnover in the upper GI tract epithelium and pancreas, improve overall gut health (Antushevich et al. 2013). Furthermore, apelin-12 has been reported to stimulate gastric acid secretion through an increase in histamine release and synthesis from gastric mucosa (Ohno et al. 2012; Huang et al. 2019).

Anastomosis promotes inflammation and as a result of this, the inflammatory cytokines increase. The Apelin/Apj expression increase is responsible for reducing the inflammatory response. Our hypothesis is that, due to ozone therapy, reduced inflammatory response and parallel to this the reduced Apelin/Apj expression is expected.

In this context, it has not been investigated whether an interaction exists between 03 treatment and apelin in the colon of peritonitis in rats. This present study aimed to evaluate the expressions of apelin and APJ with the effects of ozone therapy in a rat peritonitis model.

\section{Materials And Methods}

The experimental procedures were reviewed, approved by the Akdeniz University Local Committee on Animal Research Ethics (2014.09.18). Eighteen male Wistar albino rats (six rats in each group) weighing $250-300 \mathrm{gr}$ were used in this study. The rats were maintained at a constant temperature $\left(22^{\circ} \mathrm{C}\right)$ with a $12-$ $\mathrm{h}$ light-dark cycle and randomly divided into three groups.

\section{Experimental design and study groups}

Group 1 was the control group receiving only physiological saline. Sepsis had performed with cecal punctuation in groups 2 and 3 . Group 2 was the cecal punctuation and colonic anastomoses group. Group 3 was the group of cecal punctuation and colonic anastomoses that receive a treatment of $1 \mathrm{mg} / \mathrm{kg} /$ day at a dose of $25 \mathrm{mcg} / \mathrm{ml}$ for three weeks with medical ozone (OM-302, Ozone generator, Sedecal, Spain). At the end of the twenty-second day, the rats were sacrificed with decapitation, and the colonic samples were obtained. As previously reported, the colonic anastomoses burst pressures were measured and recorded (Cakir et al. 2016). Histopathological analysis was analyzed under a light microscope by an expert. None of the animals died during these procedures (Cakir et al. 2016).

After six hours of fasting, anesthesia was provided by intraperitoneal injection using ketamine HCL (75 $\mathrm{mg} / \mathrm{kg}$ ) (Ketasol $\circledast$, Richterpharma Ag, Wels Austria) and xylazine hydrochloride (10 mg/kg) (Rompun, Bayer, Istanbul, Turkey). Antisepsis of the incisional was provided with povidone-iodine. Then a midline laparotomy was performed using 15 numbered surgical scalpel. The abdomen in the control group was 
closed single layer with a 3/0 silk suture. In the other two groups, the colonic segment isolated was resected at the midpoint and anastomosis end to end with 6/0 polypropylene suture. After the smooth ending of anesthesia had been free access to oral feeding on the first postoperative day. The rats received postoperative analgesia with acetaminophen (Paracetamol; Sigma-Aldrich Chemistry, Steinheim, Germany) in a dose of $50 \mathrm{mg} / \mathrm{kg} / \mathrm{d}$ by oral gavage. The same midline incision had opened in all the groups three weeks later. After the sacrification of rats by decapitation tissue was obtained from a section of $10 \mathrm{~cm}$ of the colon segment (Cakir et al. 2016).

\section{Histopathological analysis}

Five-micrometer-thick sections of formalin-fixed paraffin-embedded colon tissues were dried overnight at $56{ }^{\circ} \mathrm{C}$. The samples were de-paraffinized in xylene at room temperature for $10 \mathrm{~min}$ and rehydrated with a graded ethanol series and then washed in distilled water. Antigen retrieval was performed by boiling the slides in citrate buffer $(\mathrm{pH} 6.0,0.01 \mathrm{M})$ in a microwave oven at $750 \mathrm{~W}$ for $7 \mathrm{~min}$. The sections were washed 3 times in PBS (pH:7.4) for 15 min each. Endogenous peroxidase activity was blocked by incubating the sections with $3 \% \mathrm{H}_{2} \mathrm{O}_{2}$ for $15 \mathrm{~min}$. Then, the slides were incubated with blocking serum (UV blocker Thermo Ultra UV Block, labvisio Corporation Ta-125UB) for 7 min to block non-specific immunoglobulin binding. The sections were incubated with primary antibody apelin (NBP1-07130; Novus Biologicals, Littleton, CO, USA) rabbit polyclonal at dilution of 1:300 and primer antibody APJ (bs-2430R; Bioss, Woburn, MA, USA) rabbit polyclonal at 1:500 overnight at $4{ }^{\circ} \mathrm{C}$. Subsequently, the sections were incubated with a biotinylated anti-mouse secondary antibody (Vector Lab. Inc., Burlingame, CA, USA) at 1:400 dilution for $45 \mathrm{~min}$ at room temperature. The sections were overlaid with peroxidase-labeled streptavidin (\#85-9043, Invitrogen) for 10 min and followed by rinsing in PBS for 15 min.

Diaminobenzidine (DAB, Vector, Burlingame, CA, USA) was added as a substrate and the sections were observed until they simultaneously turned brown. Finally, the sections were counter stained with hematoxylin and examined with Axioplan light microscope (Zeiss,Germany) and photographed. The antiapelin and APJ immunostaining was quantified as the average percentage of Apelin-staining and APJstaining areas by using ImageJ soft-ware.

\section{SDS-polyacrylamide gel electrophoresis and western blotting}

Tissues previously obtained and stored at $-80^{\circ} \mathrm{C}$ were removed to prepare lysate. Control and experimental groups were separated and taken to tubes. After mechanically disintegrating, lysis buffer and protease inhibitor cocktail were added. Then the samples were dissected with the help of a sonicator and centrifuged at $15000 \mathrm{rpm}$ and $+4^{\circ} \mathrm{C}$ for 10 minutes. The resulting supernatant was removed to $-20^{\circ} \mathrm{C}$. Protein measurement was performed on the obtained lysates the next day using a BCA kit. 
In order to perform immunoblot analysis of Anti-apelin and APJ; samples were subjected to SDS polyacrylamide gel electrophoresis and then were transferred onto nitrocellulose membranes (Amersham Pharmacia, Piscataway, NJ, USA) in a buffer containing $0.2 \mathrm{~mol} / \mathrm{I}$ glycine, $25 \mathrm{mMTris}$ and $20 \%$ methanol overnight. The membranes were blocked for $1 \mathrm{~h}$ with $5 \%$ non-fat dry milk (BioRad, Hercules, CA, USA) and $0.1 \%$ Tween 20 (Sigma-Aldrich Co. LLC, Steinheim, Germany) in 0.14 mol/I Tris-buffered saline (TBS) $\mathrm{pH}: 7.2-7.4$ at $4{ }^{\circ} \mathrm{C}$. Blotting membranes were incubated overnight at $4{ }^{\circ} \mathrm{C}$ with rabbit polyclonal primer antibodies apelin (NBP1-07130; Novus Biologicals, Littleton, CO, USA) at 1:1500 dilution and rabbit polyclonal primer antibody APJ (bs-2430R; Bioss, Woburn, MA, USA) at 1:1500 dilution in a humidified chamber. After washing steps, the membranes were further incubated with the seconder antibody (Vector; PI-1000 Goat Anti Rabbit) diluted 1:5000 for $1 \mathrm{~h}$ at room temperature. Immunolabeling was visualized using the chemiluminescence based Super Signal ECL HRP Substrate System (Thermo Scientific, Lot\#QI218481) and the membranes were exposed to Hyperfilm (Amersham Hyperfilm ECL, UK, Cat.no:28906838). Beta-actin antibody (Cell Signaling Technology; 13E5; 1/3000) was used as an internal control for each blotting in order to confirm the equal loading of the samples. The bands were quantified using NIH image analysis software (Image J Version 1.36b, National Institutes of Health, Bethesda, MD, USA).

\section{Statistical analysis}

Statistical analysis was performed by GraphPad Prism 3.0 (GraphPad Software, San Diego, USA). The data were expressed as mean \pm standard error of the mean (SEM). Group comparisons were performed with the analysis of variance (ANOVA) followed by Tukey's tests. The data related to immunohistochemical staining intensity and bands of western blotting of the groups and $\mathrm{J}$ image analysis was compared with the Student's t-test, using Sigma Plot 11.0 (Systat Software, USA). The $p<0.05$ between the groups was considered statistically significant.

\section{Results}

\section{Apelin and APJ immunoreactivity in the colon}

The Apelin and APJ were investigated in colonic anastomosis with ozone therapy. Mucosal glands and lamina propria were immunolabelled for both apelin and APJ in colonic sections. However, the APJ immunoreactivity was intensely observed in secretions of the mucosal glands of anastomosis group (Fig 1F). The Apelin and APJ immunoreactivities in colonic sections were quantified. The intensities of Apelin staining significantly decreased in the ozone therapy group by comparison with the control $(p<0,01)$ while significantly is lower in the ozone therapy group by comparison with the anastomosis $(p<0,001)$ (Fig 11$)$. The intensities of Apelin staining similar in control and anastomosis groups (Fig 1I). The intensities of APJ staining was not significant in the ozone therapy and anastomosis groups by comparison with the control. However, the intensities of staining was lower significantly in the ozone therapy group by comparison with the anastomosis $(\mathrm{p}<0,01)$ (Fig $1 \mathrm{~J})$. 


\section{The protein expression of Apelin and APJ in colon}

The Apelin and APJ was investigated in colon anastomosis with ozone therapy at the protein level as well (Fig 2A). The Apelin $(p<0,01)$ (Fig 2B) and APJ ( $p<0.05)$ (Fig 2C) protein levels increased significantly in the anastomosis group by comparison with the control group. Both the Apelin $(p<0,05)(F i g 2 B)$ and APJ $(\mathrm{p}<0.05)(\mathrm{Fig} 2 \mathrm{C})$ protein levels decreased significantly in the ozone therapy group by comparison with the control group. In addition, the protein levels were decreased significantly in the ozone therapy group by comparison with the anastomosis group for apelin and APJ respectively $(p<0,0001, p<0,001)$ (Figure 2 $B, C)$.

\section{Discussion}

In the present study, with the anastomosis in rats by creating investigated of the ozone therapy Apelin and APJ expression on the effect. Our results showed that the apelin and APJ act in anastomosis-derived inflammation. Also, ozone therapy mediated apelin and APJ.

Apelin and its receptor expressed intensely in the colon mucosal glands. This situation showed that apelin and its receptor can play a role in colonic secretion.

The previous studies report the role of apelin/APJ in the control and regulation of various functions in the gastrointestinal system ( $\mathrm{Lv}$ et al. 2013). Apelin, known to be expressed by adipose tissue, was strongly inhibited in the fasting state of subsequently recovered by refeeding (Boucher et al. 2005). In a human study, mean serum apelin-36 and apelin-12 concentrations, decreased in patients with anorexia nervosa with depletion fat tissue according to control (Ziora et al. 2010).

The apelin is known to be expressed in various tissues (heart, brain, lung, gastrointestinal system). The apelin expression was shown as high levels in the stomach fundus while low levels in the intestine with Northern analysis. Also, the glandular epithelium of the rat and human stomach was shown to include being the high levels of the apelin peptide with immunohistochemical. The abundant apelin peptide staining in the stomach mucosa suggests that being produced by various gastric mucosa cell types (Wang et al. 2004). The presence of apelin was demonstrated in some gastric epithelial cells. The researches indicated that apelin produced by both gastric exocrine and endocrine cells. Also, apelin expression detected that in mucous neck cells, parietal cells and chief cells. Apelin is not found in parietal neck cells, but apelin staining is present at the top of the gastric glands (Susaki et al. 2005). The expression levels of apelin and APJ, in the rat and mouse stomach, duodenum and colon, are at maximal or near maximal levels at birth while decline precipitously postnatally. Also, the apelin expression levels, is different in the immunohistochemical staining when compared to the profile of APJ protein production in the postnatal GI tract. The APJ immunostaining intensity is strong that in the surface epithelium, goblet cells and smooth muscle layer of gastrointestinal tract and it is compatible with high APJ expression levels in neonates. However, the APJ staining intensity in the surface epithelium and goblet cells decrease as rat pups mature while it is observed in the smooth muscle cells of the gastrointestinal tract during the 
entire lifespan of the rat (Wang et al. 2009). Additionally, in-vivo and in-vitro studies demonstrated that hypoxia increases the APJ expression. The acute hypoxia, in the rat pups, increases both colonic APJ mRNA levels and hepatic, stomach-duodenal, and colonic apelin mRNA levels. Also, colonic APJ mRNA levels further increase with apelin treatment during hypoxia. This data demonstrates that the colonic apelin expression increase with hypoxia or experimental colitis and colonic epithelial proliferation reduces (Han et al. 2008).

Apelin has therapeutic effects on some diseases (acute kidney, hepatic and lung injury, hypertension, gastrointestinal tract inflammation), and, as a proinflammatory factor, can stimulate inflammatory cytokine expression (Antushevich and Wojcik 2018). In a published study, it was shown that apelin expression is positively correlated with the advent of inflammation in the body. Ge et al. (2018) observed that the adipokines have been implicated between mesenteric adipose tissue and lymphatic vessels play important role in Crohn's disease. The apelin defined as an adipokine has been high expressed in mesenteric adipose tissue of Crohn's disease compared to the control group. The compared between groups of disease activity index, showed decrease of disease activity index in apelin-treated group after in the chronic colitis model (mice) the apelin-13 application as systemic. Also, in the colonic tissue of the apelin-treated group was a significantly decreased expression of TNF-a, IL-1 $\beta$, and IL-6 mRNA compared to the untreated group.

Previous studies have shown the effect of apelin-13 on gastrointestinal motility (Yang et al. 2010; Lv et al. 2011). Intracerebroventricular administration of Apelin-13 in mice has been shown to dose-dependently delay gastric emptying and gastrointestinal transit (Lv et al. 2011), inhibit distal colonic passage, fecal pellet exit, and bead expulsion (Yang et al. 2010).

The main molecular mechanisms underlying the proven therapeutic effects of ozone therapy have been reported as hypoxia-inducible factor-1a, nuclear factor of activated T cells, nuclear factor erythroid 2related factor 2-antioxidant response element, and activated protein-1 pathways. These molecular pathways lead to various mechanisms such as up-regulation of endogenous antioxidant systems, activation of immune functions, and suppression of inflammatory processes (Braidy et al. 2018). It is also reported that ozone is an effective therapeutic in the treatment of fibromyalgia, which has symptoms such as non-restorative sleep, chronic fatigue, anxiety, widespread severe pain, and depressive symptoms (Tirelli et al. 2019). Laboratory studies have shown that 03 plays a role in both as an antioxidant and modulations of the vascular, hematological, and immune systems. On the other hand, it has also been reported that 03 therapy is beneficial in cardiovascular, subcutaneous tissue, peripheral vascular disease, neurological, head and neck, orthopedic, gastrointestinal, and genitourinary pathologies (Smith et al. 2017).

O3 disrupts the self-sustaining cycle of the inflammatory cascade, altering the breakdown of arachidonic acid in inflammatory prostaglandins. 03 is involved in the activation of immune cells and has been shown to induce the production of cytokines (Di Mauro et al. 2019; Felder-Puig et al. 2009; Manoto et al. 2018). Finally, a previous study on ozone therapy showed that it effectively reduced inflammation 
through the reduction of pro-inflammatory cytokines and activation of the IL-10 anti-inflammatory cytokine (Tartari et al. 2020).

\section{Conclusions}

Consequently, our study showed that apelin and its receptor were localized widely in colon mucosal glands of control tissues, and the dramatic increase of Apelin/APJ levels after anastomosis was shown to have an active role in the anastomosis mechanism and accordingly in inflammation. Interestingly, the decrease of apelin and APJ levels after ozone therapy showed that the application of ozone can be a treatment option for anastomosis. We can speculate that ozone therapy plays a therapeutic role by decreasing apelin and APJ levels in inflammation observed by anastomosis-induction. Further molecular techniques would benefit to clarify the mechanisms controlling the Apelin-APJ system and inflammatory processes which is relieved by ozone therapy.

\section{Declarations}

-Ethics approval and consent to participate (Human Ethics, Animal Ethics or Plant Ethics): The experimental procedures were reviewed, approved by the Akdeniz University Local Committee on Animal Research Ethics (2014.09.18).

-Consent for publication: All authors give consent for publication.

-Availability of data and materials: The data that support the findings of this study are available on reasonable request.

-Competing interests: No conflict of interest was declared by the authors.

-Funding: Partial financial support was received from The Research Foundation of Akdeniz University.

-Authors' contributions: Leyla Kilinc, Sema Avcl, Tugrul Cakir designed the study.

Leyla Kilinc, Sema Avcl, Tugrul Cakir, Arif Aslaner, and Hakan Soylu collected the data, designed and developed the database, carried out data analyses, and produced the initial draft of the manuscript.

Leyla Kilinc, Gokhan Akkoyunlu and Ismail Ustunel, contributed to drafting the manuscript.

All authors participated in the revised manuscript, and have read and approved the final submitted manuscript.

-Acknowledgements: Not Applicable

\section{References}


1. Antushevich, H., B. Pawlina, M. Kapica, A. Krawczynska, A. P. Herman, A. Kuwahara, I. Kato, and R. Zabielski. 2013. Influence of fundectomy and intraperitoneal or intragastric administration of apelin on apoptosis, mitosis, and DNA repair enzyme OGG1,2 expression in adult rats gastrointestinal tract and pancreas. J Physiol Pharmacol 64 (4): 423-428.

2. Antushevich, H., and M. Wojcik. 2018. Review: Apelin in disease. Clin Chim Acta 483: 241-248. 10.1016/j.cca.2018.05.012.

3. Aslaner, A., T. Cakir, S. O. Tekeli, S. Avci, U. Dogan, F. Tekeli, H. Soylu, C. Akyuz, S. Koc, I. Ustunel, and N. Yilmaz. 2016. Medical ozone treatment ameliorates the acute distal colitis in rat. Acta Cir Bras 31 (4): 256-263. 10.1590/S0102-865020160040000006.

4. Boucher, J., B. Masri, D. Daviaud, S. Gesta, C. Guigne, A. Mazzucotelli, I. Castan-Laurell, I. Tack, B. Knibiehler, C. Carpene, Y. Audigier, J. S. Saulnier-Blache, and P. Valet. 2005. Apelin, a newly identified adipokine up-regulated by insulin and obesity. Endocrinology 146 (4):1764-1771. 10.1210/en.2004 - 1427.

5. Braidy, N., M. Izadi, A. Sureda, N. Jonaidi-Jafari, A. Banki, S. F. Nabavi, and S. M. Nabavi. 2018. Therapeutic relevance of ozone therapy in degenerative diseases: Focus on diabetes and spinal pain. J Cell Physiol 233 (4): 2705-2714. 10.1002/jcp.26044.

6. Bulbul, M., O. Sinen, I. Birsen, and V. Izgut-Uysal. 2017. Peripheral apelin-13 administration inhibits gastrointestinal motor functions in rats: The role of cholecystokinin through CCK1 receptor-mediated pathway. Neuropeptides 63: 91-97. 10.1016/j.npep.2016.12.001.

7. Bulynin, V. I., and A. A. Glukhov. 1999. [Treatment of peritonitis using ozone and hydropressive technology]. Khirurgiia (Mosk) (7):9-11.

8. Cakir, T., A. Aslaner, S. O. Tekeli, S. Avci, U. Dogan, F. Tekeli, H. Soylu, C. Akyuz, S. Koc, I. Ustunel, and N. Yilmaz. 2016. Effect of ozone on colon anastomoses in rat peritonitis model. Acta Cir Bras 31 (2): 111-118. 10.1590/S0102-865020160020000005.

9. Castan-Laurell, I., C. Dray, C. Attane, T. Duparc, C. Knauf, and P. Valet. 2011. Apelin, diabetes, and obesity. Endocrine 40 (1): 1-9. 10.1007/s12020-011-9507-9.

10. Di Mauro, R., G. Cantarella, R. Bernardini, M. Di Rosa, I. Barbagallo, A. Distefano, L. Longhitano, N. Vicario, D. Nicolosi, G. Lazzarino, D. Tibullo, M. E. Gulino, M. Spampinato, R. Avola, and G. Li Volti. 2019. The Biochemical and Pharmacological Properties of Ozone: The Smell of Protection in Acute and Chronic Diseases. Int J Mol Sci 20 (3). 10.3390/ijms20030634.

11. Erginel, B., T. Erginel, B. Aksoy, and A. I. Dokucu. 2014. Effect of Ozone Therapy (OT) on Healing of Colonic Anastomosis in a Rat Model of Peritonitis. Balkan Med J 31 (3): 249-253. 10.5152/balkanmedj.2014.13215.

12. Felder-Puig, R., M. Gyimesi, T. Mittermayr, and S. Geiger-Gritsch. 2009. [Chemonucleolysis and intradiscal electrothermal therapy: what is the current evidence?]. Rofo 181 (10): 936-944. 10.1055/s-0028-1109573.

13. Ge, Y., Y. Li, Q. Chen, W. Zhu, L. Zuo, Z. Guo, J. Gong, L. Cao, L. Gu, and J. Li. 2018. Adipokine apelin ameliorates chronic colitis in II-10(-/-) mice by promoting intestinal lymphatic functions. Biochem 
Pharmacol 148: 202-212. 10.1016/j.bcp.2018.01.011.

14. Han, S., G. Wang, X. Qi, H. M. Lee, E. W. Englander, and G. H. Greeley Jr. 2008. A possible role for hypoxia-induced apelin expression in enteric cell proliferation. Am J Physiol Regul Integr Comp Physiol 294 (6): R1832-R1839. 10.1152/ajpregu.00083.2008.

15. Huang, Z., X. Luo, M. Liu, and L. Chen. 2019. Function and regulation of apelin/APJ system in digestive physiology and pathology. J Cell Physiol 234 (6): 7796-7810. 10.1002/jcp.27720.

16. Khalili, T. M., R. A. Navarro, Y. Middleton, and D. R. Margulies. 2001. Early postoperative enteral feeding increases anastomotic strength in a peritonitis model. Am J Surg 182 (6): 621-624. 10.1016/s0002-9610(01)00818-2.

17. Lv, D., L. Li, Q. Lu, Y. Li, F. Xie, H. Li, J. Cao, M. Liu, D. Wu, L. He, and L. Chen. 2016. PAK1-cofilin phosphorylation mediates human lung adenocarcinoma cells migration induced by apelin-13. Clin Exp Pharmacol Physiol 43 (5): 569-579. 10.1111/1440-1681.12563.

18. Lv, S. Y., Y. J. Yang, and Q. Chen. 2013. Regulation of feeding behavior, gastrointestinal function and fluid homeostasis by apelin. Peptides 44: 87-92. 10.1016/j.peptides.2013.03.024.

19. Lv, S. Y., Y. J. Yang, Y. J. Qin, W. Xiong, and Q. Chen. 2011. Effect of centrally administered apelin-13 on gastric emptying and gastrointestinal transit in mice. Peptides 32 (5): 978-982. 10.1016/j.peptides.2011.01.023.

20. Manoto, S. L., M. J. Maepa, and S. K. Motaung. 2018. Medical ozone therapy as a potential treatment modality for regeneration of damaged articular cartilage in osteoarthritis. Saudi J Biol Sci 25 (4): 672-679. 10.1016/j.sjbs.2016.02.002.

21. Ohno, S., K. Yakabi, S. Ro, M. Ochiai, T. Onouchi, T. Sakurada, H. Takabayashi, S. Ishida, and K. Takayama. 2012. Apelin-12 stimulates acid secretion through an increase of histamine release in rat stomachs. Regul Pept 174 (1-3): 71-78. 10.1016/j.regpep.2011.12.002.

22. Perez-Vilar, J., and R. L. Hill. 1999. The structure and assembly of secreted mucins. J Biol Chem 274 (45): 31751-31754. 10.1074/jbc.274.45.31751.

23. Schumacher, U., M. Duku, M. Katoh, J. Jorns, and W. J. Krause. 2004. Histochemical similarities of mucins produced by Brunner's glands and pyloric glands: A comparative study. Anat Rec A Discov Mol Cell Evol Biol 278 (2): 540-550. 10.1002/ar.a.20046.

24. Smith, N. L., A. L. Wilson, J. Gandhi, S. Vatsia, and S. A. Khan. 2017. Ozone therapy: an overview of pharmacodynamics, current research, and clinical utility. Med Gas Res 7 (3): 212-219. 10.4103/2045-9912.215752.

25. Susaki, E., G. Wang, G. Cao, H. Q. Wang, E. W. Englander, and G. H. Greeley Jr. 2005. Apelin cells in the rat stomach. Regul Pept 129 (1-3): 37-41. 10.1016/j.regpep.2005.01.013.

26. Tartari, A. P. S., F. F. Moreira, M. Pereira, E. Carraro, F. J. Cidral-Filho, A. I. Salgado, and I. I. Kerppers. 2020. Anti-inflammatory Effect of Ozone Therapy in an Experimental Model of Rheumatoid Arthritis. Inflammation 43 (3): 985-993. 10.1007/s10753-020-01184-2.

27. Tirelli, U., C. Cirrito, M. Pavanello, C. Piasentin, A. Lleshi, and R. Taibi. 2019. Ozone therapy in 65 patients with fibromyalgia: an effective therapy. Eur Rev Med Pharmacol Sci 23 (4): 1786-1788. 
10.26355/eurrev_201902_17141.

28. Veneri, F., E. Bardellini, F. Amadori, G. Conti, and A. Majorana. 2020. Efficacy of ozonized water for the treatment of erosive oral lichen planus: a randomized controlled study. Med Oral Patol Oral Cir Bucal 25 (5): e675-e682. 10.4317/medoral.23693.

29. Verburg, M., I. B. Renes, H. P. Meijer, J. A. Taminiau, H. A. Buller, A. W. Einerhand, and J. Dekker. 2000. Selective sparing of goblet cells and paneth cells in the intestine of methotrexate-treated rats. $A m \mathrm{~J}$ Physiol Gastrointest Liver Physiol 279 (5): G1037-G1047. 10.1152/ajpgi.2000.279.5.G1037.

30. Wang, G., Y. Anini, W. Wei, X. Qi, O. C. AM, T. Mochizuki, H. Q. Wang, M. R. Hellmich, E. W. Englander, and G. H. Greeley Jr. 2004. Apelin, a new enteric peptide: localization in the gastrointestinal tract, ontogeny, and stimulation of gastric cell proliferation and of cholecystokinin secretion.

Endocrinology 145 (3):1342-1348. 10.1210/en.2003 - 1116.

31. Wang, G., R. Kundu, S. Han, X. Qi, E. W. Englander, T. Quertermous, and G. H. Greeley Jr. 2009. Ontogeny of apelin and its receptor in the rodent gastrointestinal tract. Regul Pept 158 (1-3): 32-39. 10.1016/j.regpep.2009.07.016.

32. Yang, Y. J., S. Y. Lv, M. H. Xiu, N. Xu, and Q. Chen. 2010. Intracerebroventricular administration of apelin-13 inhibits distal colonic transit in mice. Peptides 31 (12): 2241-2246. 10.1016/j.peptides.2010.09.006.

33. Ziora, K., J. Oswiecimska, E. Swietochowska, D. Ziora, Z. Ostrowska, M. Stojewska, E. KlimackaNawrot, A. Dyduch, and B. Blonska-Fajfrowska. 2010. Assessment of serum apelin levels in girls with anorexia nervosa. J Clin Endocrinol Metab 95 (6):2935-2941. 10.1210/jc.2009 - 1958.

\section{Figures}




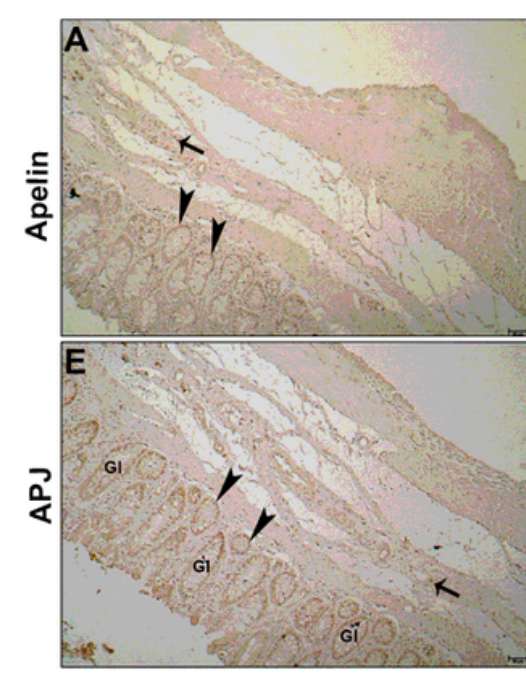

Control
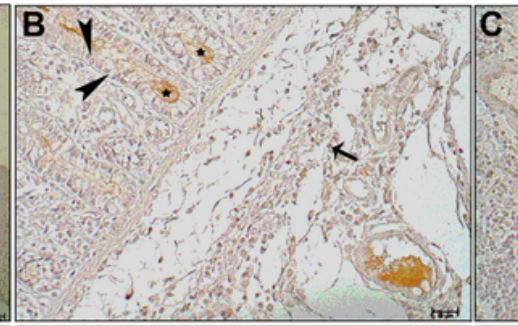

(1)

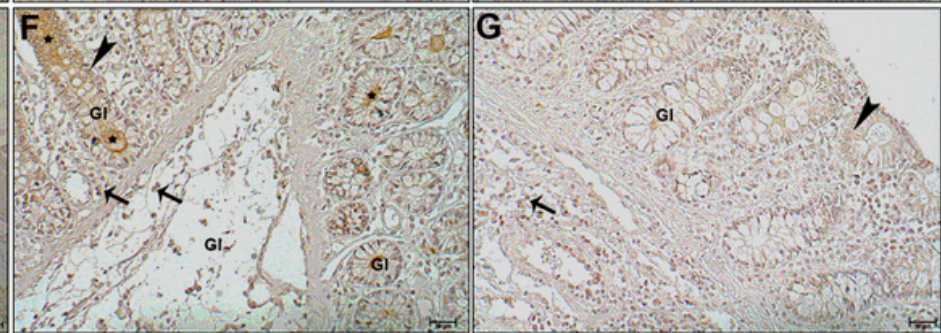

Anastomosis

Ozone therapy

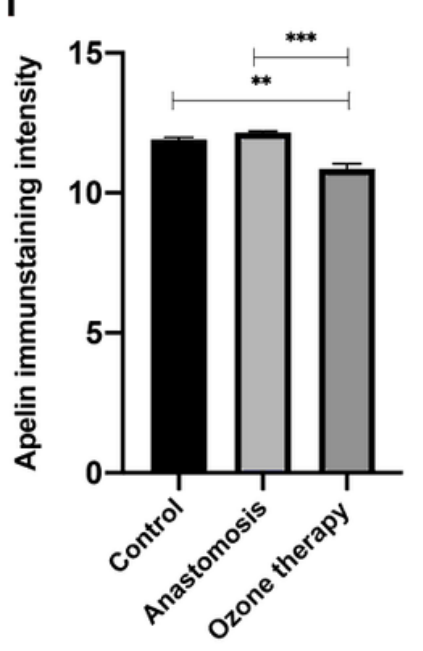

$\mathbf{J}$

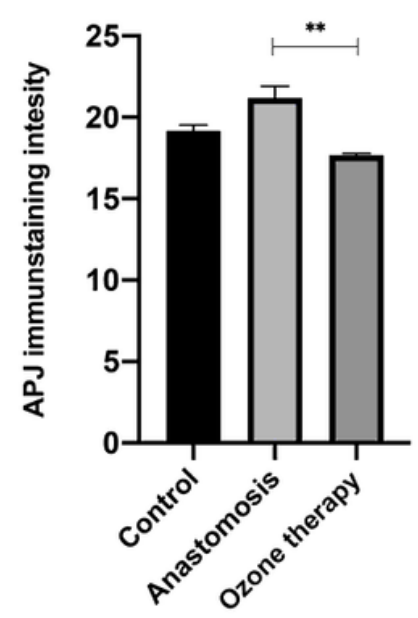

\section{Figure 1}

Immunohistochemical staining of colon sections. Apelin immunostaining intensity of the control, anastomosis, ozone therapy groups and negative control (A: x10; B: x20; C: x20; D: x40). APJ immunostaining intensity of the control, anastomosis, ozone therapy groups, and negative control (E: x10; F: x20 ; G: x20; H: x40) Arrows: connective tissue cells, arrowheads: cytoplasms of gland epithelia, asterisk: mucus secretion, Gl: gland I) Quantification of Apelin immunostaining. Apelin protein expression is significantly decreased by ozone therapy, compared to anastomosis and control groups (respectively $p<0,001, p<0,01) J$ ) Quantification of APJ immunostaining. APJ protein expression is significantly decreased by ozone therapy, compared to anastomosis groups $(p<0,01)$ 


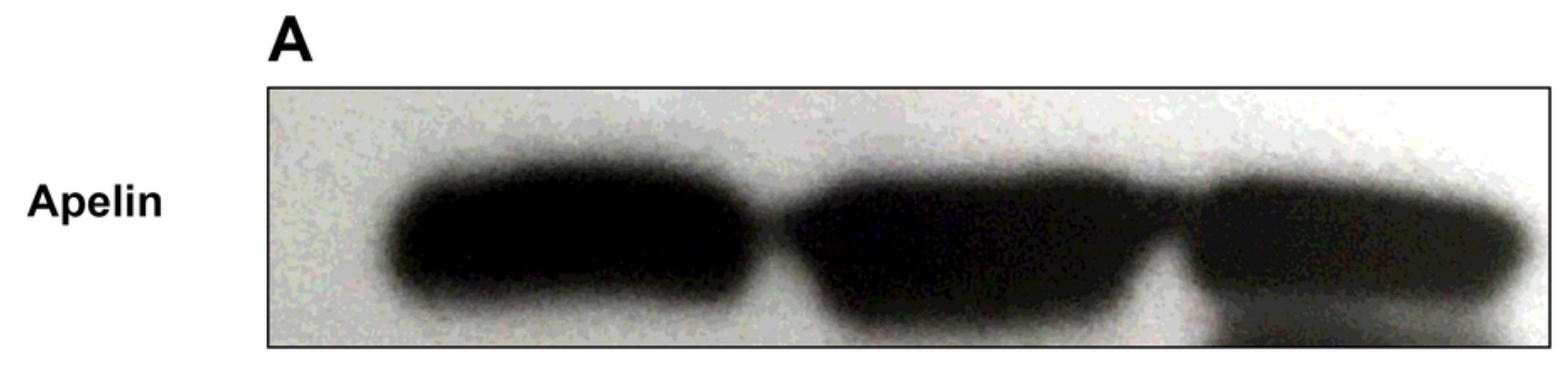

$8 \mathrm{kDa}$

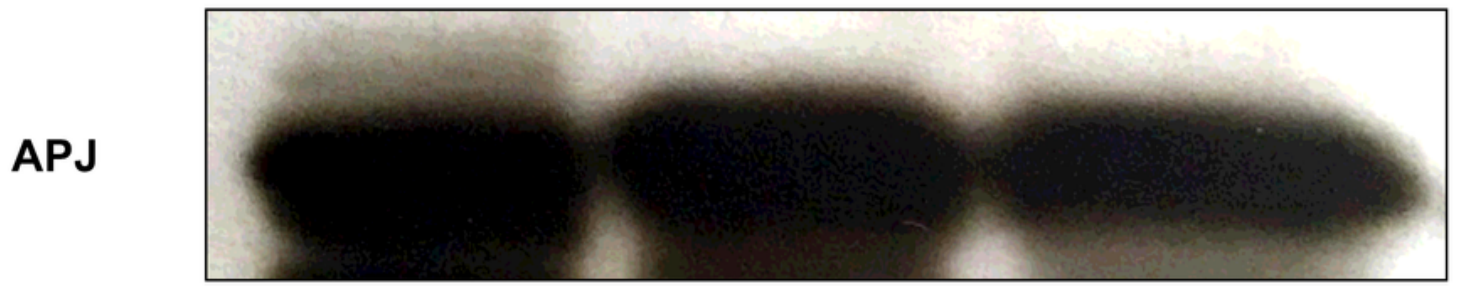

$42 \mathrm{kDa}$

\section{Beta-actin}

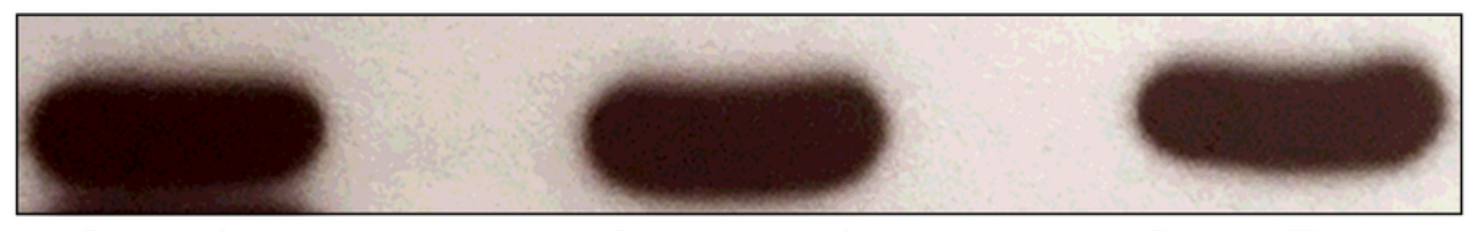

$43 \mathrm{kDa}$

Control

B

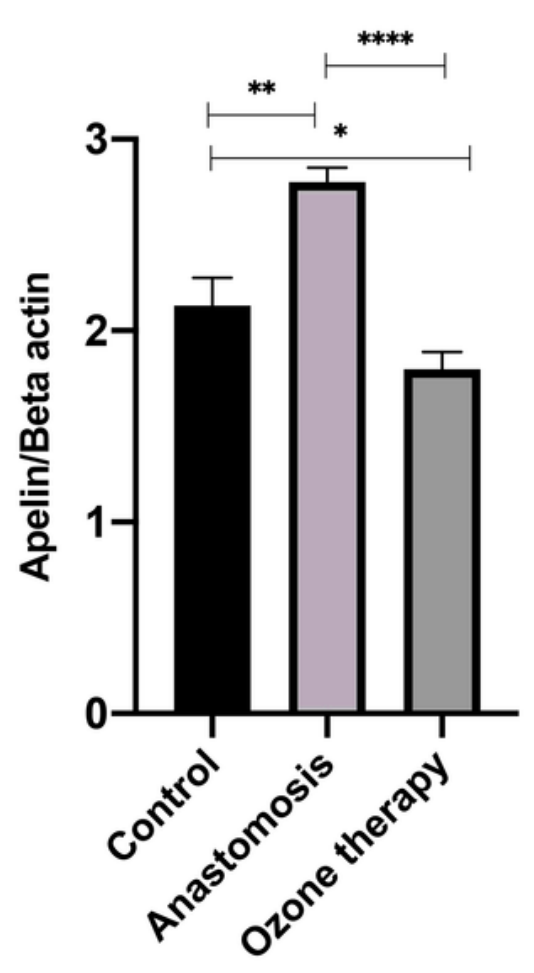

Anastomosis

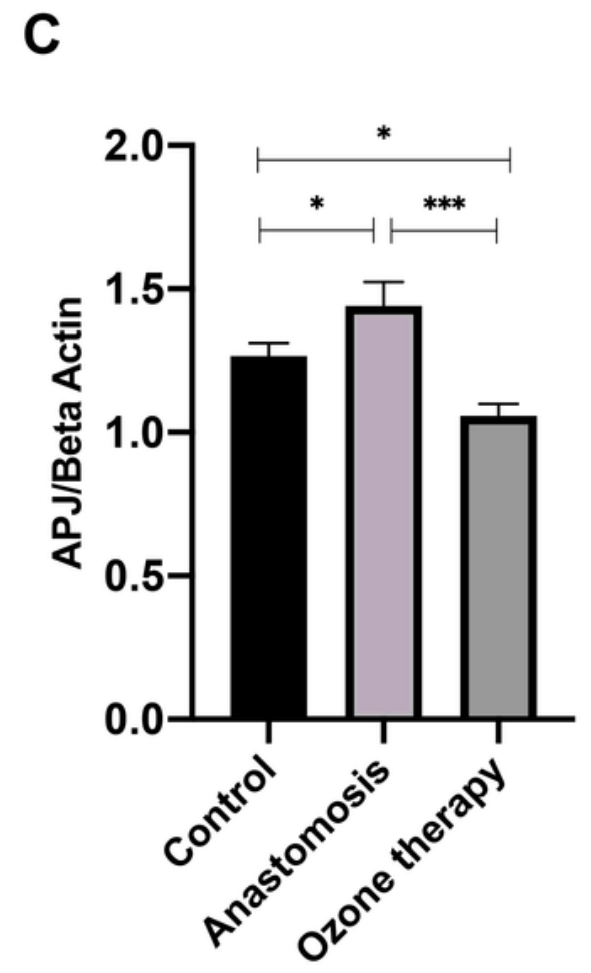

Figure 2

A) Western blot bands of apelin, APJ, and Beta-actin in control, anastomosis, and ozone therapy groups

B) Quantification of protein levels for apelin. Apelin protein level is significantly decreased by ozone therapy, compared to anastomosis and control groups (respectively $p<0,0001, p<0,05$ ) C) Quantification of protein levels for APJ. APJ protein level is significantly decreased by ozone therapy, compared to anastomosis and control groups (respectively $p<0,001, p<0,05$ ) 